\title{
THE
}

3-1-1987

\section{Critical Surface Free Energies and Universal Finite-Size Scaling Amplitudes of Three-Dimensional XY Models by Direct Monte Carlo Sampling}

\author{
K. K. Mon \\ M. P. Nightingale \\ University of Rhode Island, nightingale@uri.edu
}

Follow this and additional works at: https://digitalcommons.uri.edu/phys_facpubs

Terms of Use

All rights reserved under copyright.

\section{Citation/Publisher Attribution}

Mon, K. K., \& Nightingale, M. P. (1987). Critical surface free energies and universal finite-size scaling amplitudes of three-dimensional XY models by direct Monte Carlo sampling. Physical Review B, 35(7), 3560-3562. doi: 10.1103/PhysRevB.35.3560

Available at: http://dx.doi.org/10.1103/PhysRevB.35.3560

This Article is brought to you for free and open access by the Physics at DigitalCommons@URI. It has been accepted for inclusion in Physics Faculty Publications by an authorized administrator of DigitalCommons@URI. For more information, please contact digitalcommons-group@uri.edu. 


\title{
Critical surface free energies and universal finite-size scaling amplitudes of three-dimensional $X Y$ models by direct Monte Carlo sampling
}

\author{
K. K. Mon* and M. P. Nightingale \\ Department of Physics, University of Rhode Island, Kingston, Rhode Island 02881
}

(Received 25 June 1986)

\begin{abstract}
Direct Monte Carlo sampling is employed to obtain estimates of excess surface free energies of three-dimensional $X Y$ models at criticality. Results for simple-cubic and body-centered-cubic lattices are consistent with universality of finite-size scaling amplitudes. The results are used to estimate the magnitude of the thinning effect, mediated by the incipient long-ranged correlations, on liquid-helium films at the $\lambda$ point.
\end{abstract}

Finite-size corrections to the bulk free energy density, and excess surface free energy, measured in units of $k_{B} T$, have universal critical amplitudes. This is suggested by a variety of recent, theoretical studies. ${ }^{1-7}$ The finite-size amplitude is directly measurable, in principle. For examples, if two parallel plates are immersed in a critical system, the long-ranged correlations produce a power-law interaction between the plates. ${ }^{8}$ Alternatively, in determining the thickness of wetting layers at a critical end point, particularly in the vicinity of a tricritical point, the finite-size effect may play a dominant role. ${ }^{6}$ For twodimensional systems the amplitudes (for various boundary conditions) can be expressed in terms of the central charge, and are known in many cases. ${ }^{2,7,9}$ In three dimensions, there are (a) the exact results for the spherical model and ideal Bose gas, ${ }^{3}$ (b) the $\epsilon=1$ result obtained from Symanzik's $\epsilon$ expansion, ${ }^{1}$ (c) the Migdal-Kadanoff renormalization-group results of Refs. 6 and 7, and finally (d) the results obtained by Monte Carlo calculations for the Ising model. ${ }^{4,5}$ In this paper we report results for the three-dimensional $X Y$ model, both for the simple-cubic (sc) and body-centered-cubic (bcc) models. The critical point of this model is in the same universality class as the superfluid transition in liquid helium. Therefore, the amplitude calculated here may (depending on its relative magnitude compared to, e.g., van der Waals forces) be directly observable in the thickness of helium wetting layers at the $\lambda$ point.

We consider isotropic systems: finite rectangular prisms (with the edges along the cubic axes) of size $n_{\|} \times n_{\|} \times n_{1}$, each linear dimension expressed in units of the lattice constant $a$. Two types of boundary conditions are used: (a) periodic in all directions and (b) periodic in the $n_{\|}$directions, free otherwise.

Denote by $F$ the total dimensionless free energy of a system with boundary conditions (a), i.e., $F=-\ln (Z)$, where $Z$ is the partition function. The analog of $F$ for case (b) is denoted $F^{x}$. According to finite-size scaling these free energies are given by ${ }^{1-9}$

$$
\begin{aligned}
& F\left(n_{\|}, n_{\perp}\right) / n_{\|}^{2}=n_{\perp} f_{\infty}+n_{\perp}^{-2} \Delta^{p}+\cdots, \\
& F^{x}\left(n_{\|}, n_{\perp}\right) / n_{\|}^{2}=n_{\perp} f_{\infty}+f_{\infty}^{x}+n_{\perp}^{-2} \Delta^{f}+\cdots .
\end{aligned}
$$

Here $f_{\infty}$ is the bulk free energy (in units $k_{B} T$ ) per unit volume (in units $a^{3}$ ) and $f_{\infty}^{x}$ is twice the excess surface free energy per unit area of a free surface. Both $f_{\infty}$ and $f_{\infty}^{x}$ are defined in the thermodynamic limit of infinite $n_{\|}$ and $n_{\perp}$. Both $\Delta^{p}$ and $\Delta^{f}$ are universal functions, which depend only on the aspect ratio $n_{1} / n_{\|}$. The ellipses in Eqs. (1) indicate corrections to scaling.

The universal function we have calculated is the amplitude of the finite-size correction to $f^{x} \equiv\left(F^{x}-F\right) / n_{\|}^{2}$, which amplitude is denoted $\Delta^{-} \equiv \Delta^{f}-\Delta^{p}$. Employing a method introduced by Mon and Jasnow, ${ }^{10}$ the calculations were performed the same way as described in a previous paper, to which we refer the reader for details. ${ }^{5}$ Estimates for the critical coupling constants $K_{c}=0.4539$ (sc) and $K_{c}=0.3204$ (bcc) were taken from the literature. ${ }^{11}$

Figure 1 shows the function $\Delta^{-}\left(n_{\perp} / n_{\|}\right)$for the bcc lattice over a range of aspect ratios. For the sc lattice the calculations were done only for systems of unit aspect ratio, i.e., cubes. Within the limited accuracy of the present computation, results at this special point for the two lattices are consistent with universality. From our data we estimate $\Delta^{-}(0) \simeq 0.2 \pm 0.05$, where the error is mainly due to the uncertainty in $f_{\infty}^{x}$.

The quantity of experimental interest is the amplitude of the potential acting on the interfaces bounding a slab of, say, fluid of finite thickness in equilibrium with the same in bulk. This amplitude is $\Delta^{f}$ in the limit of vanish-

TABLE I. Summary of Monte Carlo results for three-dimensional $X Y$ models. Critical couplings were taken from Ref. 11. Note that $f_{\infty}^{x}$ is the excess surface free energy of two surfaces (statistical error in parentheses).

\begin{tabular}{lccccc}
\hline \hline & $K_{c}$ & $f_{\infty}^{x}$ & $\Delta^{-}(0)$ & $\Delta^{-}(1)$ & $\Delta^{f}(0)$ \\
\hline sc & 0.4539 & $0.076(2)$ & & $\sim 0.65$ & \\
bcc & 0.3204 & $0.165(2)$ & $\sim 0.2$ & $\sim 0.77$ & $\sim 0.05$ \\
\hline \hline
\end{tabular}




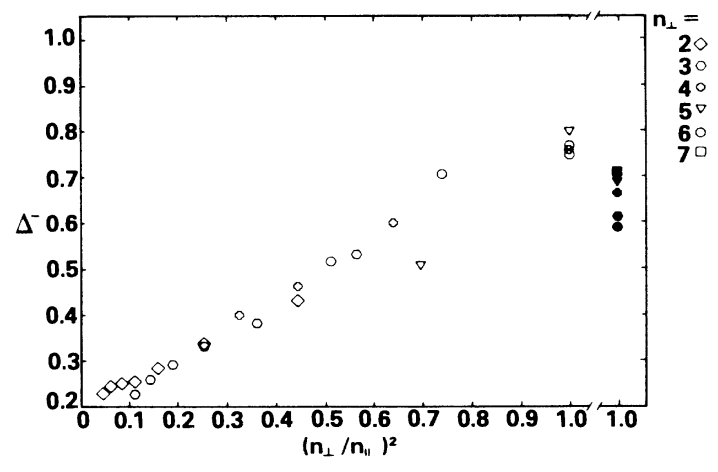

FIG. 1. Universal finite-size scaling amplitude $\Delta^{-}$, as a function of aspect ratio squared. Data for bcc and sc lattices are indicated by solid and open symbols, respectively. For the sc we have only considered the case $n_{1} / n_{\|}=1$; data for this lattice have been moved to the right for clarity. For the bcc lattice data are displayed for various aspect ratios, with $n_{||}=3,4, \ldots, 9$. Different values of $n$ have been indicated as follows: $n_{\perp}=2$ diamonds, $n_{\perp}=3$ hexagons, $n_{\perp}=4$ crosses, $n_{1}=5$ inverted triangles, $n_{1}=6$ circles, and $n_{1}=7$ squares. The excess surface free energy and the estimated error in the last digit are found by optimization of the data collapse in each case: $f_{\infty}^{x}=0.076(2)$ and $f_{\infty}^{x}=0.165$ (2) for sc and bcc lattices, respectively. The deviation of each individual data point from a smooth curve is not statistically significant.

ing aspect ratio. The amplitude $\Delta^{-}$, we calculated, can be measured in a thought experiment only. In this case the two systems in equilibrium are of the same size and differ only in boundary conditions. However, on the basis of our calculations we can make a plausible guess for $\Delta^{f}$, the experimental amplitude. One way to proceed is to assume that the relation $\Delta^{p} / \Delta^{f}=4$, which in two dimensions holds for all universality classes, is also roughly valid in three dimensions. This gives us an estimate $\Delta^{f} \simeq \Delta^{-} / 3 \simeq-0.07$. Alternatively, we can assume that the ratios of corresponding amplitudes in different universality classes in three dimensions are constant, as indeed they are in two dimensions. Since the amplitude calculated here is roughly twice as large as for the Ising model,
TABLE II. Dimensionless free energy differences $F^{x}-F$ for cubes of size $n_{1}=n_{\|}$for the sc lattice. The statistical error is estimated to be 0.005 or less in all cases.

\begin{tabular}{cc}
\hline \hline$n_{\perp}=n_{\|}$ & $F^{x}-F$ \\
\hline 3 & 0.144 \\
4 & 0.118 \\
5 & 0.103 \\
6 & 0.093 \\
7 & 0.090 \\
\hline
\end{tabular}

we find from the estimate $\Delta^{f} \simeq-0.015$ for the latter, ${ }^{6,7}$ a value of $\Delta f \simeq-0.03$ for the $X Y$ model. We tentatively conclude that $\Delta^{f}$ for the three-dimensional $X Y$ model is negative (implying a tendency to thin wetting layers) and of the order of a few hundredths, We reiterate that this amplitude is expected to be universal. This is true in particular with respect to variation of the surface interaction strength, at least below the critical value necessary to cross over from the ordinary to the extraordinary surface transition.

One would like to compare the critical point amplitude $\Delta^{f}$ with the net effect of the van der Waals forces acting on a film. The dimensionless amplitude of these forces was calculated by summing Lennard-Jones pair potentials. ${ }^{12}$ These calculations indicate for helium wetting layers at gas-liquid coexistence that the van der Waals forces have a thickening effect much stronger than the thinning due to criticality at the $\lambda$ point. However, wetting properties of liquid helium are not at all understood. While older experimental work is in agreement with the theoretical idea that helium, due to its low polarizability, should wet virtually all solids, ${ }^{13}$ some recent experiments indicate that ${ }^{4} \mathrm{He}$ does not wet gold and silver substrates at gas-liquid coexistence from below the $\lambda$ point up to the critical point. ${ }^{14}$ In yet other experiments, it is found that normal helium does not wet graphite or platinum. ${ }^{15}$ In these same experiments a wetting transition takes place at the onset of superfluidity. In view of this, even the result that the net van der Waals force acting on the gas-liquid interface tends to thicken the film is suspect, let alone the strength of the effect.

TABLE III. Dimensionless free energy differences $F^{x}-F$ for rectangular prisms of size $n_{\|} \times n_{\|} \times n_{\perp}$ for the bcc lattice. The statistical error is estimated to be of the order 0.01 in all cases.

\begin{tabular}{lllllll}
\hline$n_{\|} n_{\perp}$ & 7 & 6 & 5 & 4 & 3 & 2 \\
\hline 9 & & & & & 0.191 & 0.223 \\
8 & & & & & 0.194 & 0.225 \\
7 & 0.180 & 0.185 & 0.186 & 0.190 & 0.198 & 0.227 \\
6 & & 0.186 & 0.185 & 0.194 & 0.202 & 0.228 \\
5 & & & 0.197 & 0.202 & 0.207 & 0.235 \\
4 & & & 0.212 & 0.224 & 0.249 \\
3 & & & & 0.250 & 0.274 \\
\hline \hline
\end{tabular}


Finally, we summarized our main results in Table I. Tables II and III display the raw Monte Carlo data for the dimensionless free energy differences $F^{x}-F$ for the sc and bec models of various sizes.
It is our pleasure to acknowledge valuable discussions with Dr. J. O. Indekeu. This work was supported by the National Science Foundation, Contract No. DMR8406186 , and by a grant from the Research Corporation.
${ }^{*}$ Present address: Department of Physics and Astronomy, University of Georgia, Athens, GA 30602.

${ }^{1}$ K. Symanzik, Nucl. Phys. B 190, [FS3], 1 (1981); V. Privman and M. E. Fisher, Phys. Rev. B 30, 322 (1984); M. P. Nightingale and H. W. J. Blöte, J. Phys. A 16, L657 (1983); H. W. J. Blöte and M. P. Nightingale, Physica 134A, 274 (1985).

${ }^{2}$ H. W. J. Blöte, J. L. Cardy, and M. P. Nightingale, Phys. Rev. Lett. 56, 742 (1986).

${ }^{3}$ S. Singh and R. K. Pathria, Phys. Rev. B 31, 4483 (1985); Phys. Lett. 55, 347 (1985); Phys. Rev. B 32, 4618 (1985).

${ }^{4}$ K. K. Mon, Phys. Rev. Lett. 54, 2670 (1985).

${ }^{5}$ K. K. Mon and M. P. Nightingale, Phys. Rev. B 31, 6137 (1985). Note that in the caption of Fig. 1 of this paper $n_{\|}$ should be replaced by $n_{\perp}$; further, the lattice constant $a$ in Eq. (1) should be dropped.

${ }^{6}$ M. P. Nightingale and J. O. Indekeu, Phys. Rev. Lett. 54, 1824
(1985).

${ }^{7}$ J. O. Indekeu, M. P. Nightingale, and W. V. Wang, Phys. Rev. B 34, 330 (1986).

${ }^{8}$ M. E. Fisher and P.-G. de Gennes, C. R. Acad. Sci., Ser. B 287, 207 (1978).

${ }^{9}$ H. Au-Yang and M. E. Fisher, Phys. Rev. B 11, 3469 (1975); 21, 3956 (1980); and M. E. Fisher and H. Au-Yang, Physica 101A, 225 (1980).

${ }^{10}$ K. K. Mon and D. Jasnow, Phys. Rev. A 30, 670 (1984).

${ }^{11}$ M. Ferer, M. A. Moore, and M. Wortis, Phys. Rev. B 8, 5205 (1973).

12J. O. Indekeu (unpublished).

${ }^{13}$ E. S. Sabisky and C. H. Anderson, Phys. Rev. A 7, 790 (1973).

${ }^{14}$ A. D. Migone, J. Krim, and J. G. Dash, Phys. Rev. B 31, 7643 (1985).

${ }^{15}$ P. Taborek and L. Senator, Phys. Rev. Lett. 57, 218 (1986). 